
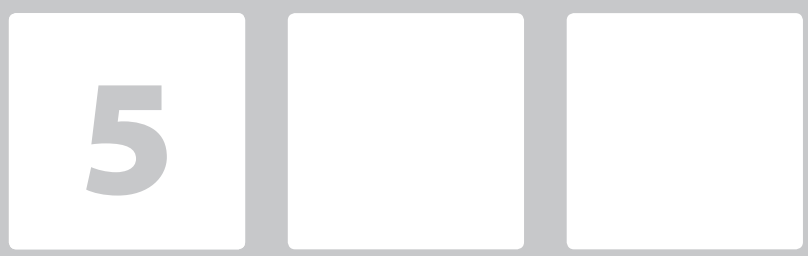

\title{
SMART CITIES E O DESENVOLVIMENTO SUSTENTÁVEL: REVISÃO DE LITERATURA E PERSPECTIVAS DE PESQUISAS FUTURAS
}

\section{Smart Cities and Sustainable Development: Literature Review and Prospects for Future Research}

\section{Denise Genari}

Dra. em Adm. Instituto Fed. de Educ., Ciência e Tecn. do Rio Grande do Sul e Univ. de Caxias do Sul. Profa do Ensino Básico, Técnico e Tecnológico. Bento Gonçalves (RS).e-mail: denisegenari@hotmail.com

\section{Luana Folchini da Costa}

Mestre em Administração. Universidade de Caxias do Sul. Psicóloga Organizacional. Caxias do Sul (RS).e-mail: luana.folchini@gmail.com

\section{Thiago Paese Savaris}

Mestre em Administração. Universidade de Caxias do Sul. Analista sênior de Remuneração - Bruning Tecnometal. Panambi (RS). e-mail:savarist@hotmail.com

\section{Janaina Macke}

Dra em Adm. Univ. de Caxias do Sul e IMED Business School. Profa. e pesquisadora. Caxias do Sul (RS).e-mail:jmacke@terra.com.br

\section{RESUMO}

O processo acelerado de urbanização da sociedade é acompanhado de desafios vinculados às esferas sociais, ambientais e econômicas. Diante desse contexto, destaca-se a emergência do tema smart cities, sendo essas consideradas espaços nos quais ocorre a utilização adequada da infraestrutura disponível, visando melhorar a eficiência econômica e política, permitindo o desenvolvimento social, cultural e urbano. Com base nessa perspectiva, o presente artigo tem como principal objetivo caracterizar os estudos que vinculam os temas smart cities e desenvolvimento sustentável, além de identificar oportunidades de pesquisas futuras. Para tanto, realizou-se uma revisão sistemática de literatura, utilizando-se o suporte do software Alceste. Os principais resultados apontam a predominância de estudos qualitativos, abordando, principalmente, a dimensão ambiental da sustentabilidade e a dimensão sistêmica vinculada às smart cities, contemplando as tecnologias da informação e da comunicação. A partir desses achados, sugere-se a realização de pesquisas futuras que considerem a dimensão social e econômica da sustentabilidade.

Palavras-chave: Smart Cities. Desenvolvimento Sustentável. Sustentabilidade. Revisão Sistemática. Software Alceste.

\section{ABSTRACT}

The accelerated process of urbanization of society is accompanied by challenges associated with social, environmental and economic spheres. In this context, emphasis is placed on the emergence of the smart cities theme, which means spaces where the adequate use of the available infrastructure occurs, with the objective of improving economic and political efficiency, allowing social, cultural and urban development. Based on this perspective, the main objective of this article is to characterize the studies that link the themes of smart cities and sustainable development, in addition to identifying future research opportunities. To this end, a systematic review of the literature was carried out using the Alceste software support. The main results point to the predominance of studies based on qualitative research, primarily addressing the environmental dimension of sustainability and the systemic dimension related to smart cities, including information and communication technologies. Based on these findings, it is recommended that future research should be carried out also taking into consideration the social and economic dimension of sustainability.

Keywords: Smart Cities. Sustainable Development. Sustainability. Systematic Review. Alceste Software. 


\section{INTRODUÇÃO}

As cidades surgiram a partir da necessidade dos indivíduos de obter segurança e de conviver de forma coletiva, com maior acesso a recursos e níveis mais elevados de qualidade de vida (ZUBIZARRETA; SERAVALLI; ARRIZABALAGA, 2015). Em convergência, as smart cities são definidas como as cidades com desempenho satisfatório em termos de resultados econômicos, de governança, de mobilidade e de meio ambiente, alinhado a cidadãos com comportamento consciente e independente (GIFFINGER et al., 2007).

$\mathrm{Na}$ atualidade, as cidades se apresentam como sistemas complexos, que são caracterizados pela conexão entre cidadãos e organizações, por diferentes modos de transportes, de redes de comunicação e de serviços (HARRISON; DONNELLY, 2011; BATTY et al., 2012; NEIROTTI, et al., 2014). Nesse sentido, as cidades são consideradas símbolos de riqueza, inovação, acessibilidade a serviços e a sistemas educacionais e oportunidades de emprego, sendo que esses fatores promovem um fenômeno contínuo de migração da população da zona rural para as áreas urbanas (CORSINI; RIZZI; FREY, 2016). O crescimento populacional e da urbanização fomentam uma variedade de desafios técnicos, sociais, econômicos e corporativos, que podem comprometer o desenvolvimento sustentável nas esferas econômica, social e ambiental das cidades (NEIROTTI, et al., 2014; MEIJER; BOLÍVAR, 2016).

O tema desenvolvimento sustentável tem se apresentado como relevante, principalmente nas últimas décadas, em virtude do rápido esgotamento dos recursos naturais, das preocupações com as disparidades de riqueza na coletividade e da importância da responsabilidade social corporativa (DYLLICK; HOCKERTS, 2002; LINNENLUECKE; GRIFFITHS, 2010; DAO; LANGELLA; CARBO, 2011; GUERCI; SHANI; SOLARI, 2013). Além disso, as atuais mudanças climáticas, as discussões acerca das consequências da globalização, a crise nos mercados financeiros e a necessidade de desenvolvimento de uma orientação, por parte das instituições, para seus stakeholders, reforça a importância do tema (JAMALI, 2008; ZINK, 2014).

Em convergência com esse cenário, percebe-se, no âmbito organizacional e acadêmico, o crescente interesse por estudos que enfoquem a sustentabilidade e o desenvolvimento sustentável (LINNENLUECKE; GRIFFITHS, 2010; DYLLICK; MUFF, 2015). Embora o número de pesquisas tenha aumentado nos últimos anos (ROCA; SEARCY, 2012; LEAL FILHO et al., 2018), ainda existem divergências em termos conceituais (GLAVIČ; LUKMAN, 2007; JOHNSTON et al., 2007; CLARO; CLARO; AMÂNCIO, 2008; EHNERT, 2009; BULLER; MCEVOY, 2016), uma vez que a sustentabilidade se apresenta como um componente de várias disciplinas científicas. Ademais, o termo eventualmente é empregado apenas como uma expressão teórica e com cunho político ou como uma "expressão da moda", fatos que desvirtuam o verdadeiro propósito da sustentabilidade (LEAL FILHO, 2000).

Também verifica-se, no campo acadêmico e institucional, um crescente interesse e a ampliação dos estudos que abordam o tema smart cities (CARAGLIU; DEL BO; NIJKAMP , 2011; LEE; LEE, 2014; NEIROTTI, et al., 2014; CORSINI; RIZZI; FREY, 2016; MASSANA, et al., 2017). No entanto, ainda percebe-se a oportunidade de ampliação dessas pesquisas, uma vez que os conhecimentos nesse contexto ainda são fragmentados e vinculados, em muitas situações, a conceitos vagos ou imprecisos (SÁNCHEZ et al., 2013; LEE; HANCOCK; HU, 2014; ALBINO; BERARDI; DANGELICO, 2015; ANGELIDOU, 2015; PAPA et al., 2015; CORSINI; RIZZI; FREY, 2016; MEIJER; BOLÍVAR, 2016; MASSANA, et al., 2017).

A multiplicidade conceitual está evidenciada em estudos que se concentram em diferentes tópicos: questões ambientais, com ênfase no uso eficiente dos recursos naturais e consumo de energia; condições socioeconômicas, com destaque para a relevância do capital social e do capital humano; potencial das tecnologias da informação e da comunicação (TICs), para a melhoria dos processos de tomada de decisões e para o empoderamento das comunidades locais (PAPA et al., 2015). Logo, percebe-se que os estudos sobre smart cities necessitam de uma abordagem mais holística e integrativa, ao se considerar que muitas pesquisas enfocam, de maneira segregada, os tópicos anteriormente citados (ALAWADHI et al., 2012; ANTTIROIKO; VALKAMA; BAILEY, 2014; CORSINI; RIZZI; FREY, 2016; MEIJER; BOLÍVAR, 2016).

A literatura assinala que a emergência do tema smart cities também está relacionada às perspectivas 
do desenvolvimento sustentável (DATTA, 2015; HÖJER; WANGEL, 2015; STRATIGEA; PAPADOPOULOU; PANAGIOTOPOULOU, 2015), por intermédio da integração dos aspectos tecnológicos, dos sistemas sociais e das preocupações com o meio ambiente (ANTTIROIKO; VALKAMA; BAILEY, 2014). Diante desse contexto, destaca-se que o presente artigo tem por objetivo caracterizar os estudos que vinculam os temas smart cities e desenvolvimento sustentável, além de identificar oportunidades de pesquisas futuras. Para tanto, desenvolveu-se uma revisão sistemática de literatura, considerando-se que esse método possibilita sintetizar um volume considerável de informações, objetivando a identificação das principais características sobre determinados temas (PETTICREW; ROBERTS, 2006).

Para o atendimento desse objetivo, nas próximas seções, serão apresentados os principais conceitos vinculados aos temas smart cities e desenvolvimento sustentável, bem como os procedimentos metodológicos adotados para o estudo, a análise e discussão dos principais resultados verificados.

\section{SMART CITIES}

As cidades podem ser consideradas o futuro da humanidade. Tal afirmação, segundo Harrison e Donnelly (2011), se consolida pelo fato de que, no século XVIII, apenas 5\% da população mundial residia em uma grande cidade. Como tendência, verifica-se a inversão significativa dessa proporção, com a expectativa de que $80 \%$ da população mundial resida em cidades, até o final do século XXI, fazendo com que a população urbana supere, de forma expressiva, a rural (PAPA et al., 2015; ZUBIZARRETA; SERAVALLI; ARRIZABALAGA, 2015). Nesse contexto, identifica-se um processo acelerado de urbanização da sociedade (CARAGLIU; DEL BO; NIJKAMP, 2011; LEE; LEE, 2014; CARAGLIU et al., 2015; CASSANDRAS, 2016), acompanhado de desafios oriundos desse movimento (KUTAMI; TAKENO; IOKA, 2014; AHVENNIEMI et al., 2017; MASSANA, et al., 2017), como a poluição do ar, os congestionamentos, o aumento do consumo de energia e de recursos naturais, o crescimento da emissão de gases estufa, o aquecimento global, entre outros (ALAWADHI et al., 2012; LAZAROIU;
ROSCIA, 2012; BAKICI; ALMIRALL; WAREHAM, 2013; COHEN; AMORÓS, 2014; FERNÁNDEZ et al., 2014; PAPA et al., 2015; STÅHLBRÖST et al., 2015).

A aceleração do crescimento populacional faz emergir duas questões conflitantes (BIFULCO et al., 2016). Por um lado, verifica-se a necessidade de exploração excessiva dos recursos, o aumento da poluição e um número insuficiente de serviços disponibilizados. Por outro, existe a necessidade de implementação dos preceitos da sustentabilidade para superar essas situações críticas. Nesse sentido, embora o crescimento urbano possa ser apontado como um dos fatores de origem dos problemas ambientais, também pode-se assinalar que as cidades, pela diversidade populacional, podem se tornar centros de criatividade e de inovação que, por sua vez, apresentam a capacidade de elaborar estratégias de atenuação ou adaptação ao cenário descrito (KOURTIT; NIJKAMP, 2012; PAPA et al., 2015). Nesse contexto, os problemas associados com as aglomerações urbanas têm sido atenuados por meio da criatividade, do capital humano, da cooperação entre as partes interessadas e de ideias científicas consideradas como "smart solutions" (CARAGLIU; DEL BO; NIJKAMP, 2011).

A fim de responder aos desafios vinculados ao contexto apresentado anteriormente, destaca-se o surgimento do conceito das smart cities (CHOURABI et al., 2012), na década de 1990 (CARAGLIU; DEL BO; NIJKAMP, 2011; ALBINO; BERARDI; DANGELICO, 2015), com maior ênfase a partir de 2009 (MARSAL-LLACUNA; COLOMER-LLINÀS; MELÉNDEZ-FRIGOLA, 2015). Nessas cidades ocorrem investimentos no capital social e humano, mobilidade urbana, modernas instalações de infraestrutura de comunicação e tecnologia, crescimento econômico sustentável e alta qualidade de vida, aliados à sensata gestão dos recursos naturais, através da governança participativa (CARAGLIU; DEL BO; NIJKAMP, 2011). Para tanto, uma smart city contempla uma gestão racional dos recursos naturais, com um nível adequado de investimentos em infraestrutura de transportes e um compromisso representativo com o desenvolvimento e a utilização de produtos e serviços vinculados às modernas tecnologias da informação e da comunicação (CORSINI; RIZZI; FREY, 2016). Além disso, nas smart cities ocorre a utilização adequada da infraestrutura disponível, com o objetivo 
de melhorar a eficiência econômica e política, permitindo o desenvolvimento social, cultural e urbano (HOLLANDS, 2008).

No contexto das smart cities, o advento tecnológico tornou-se um elemento-chave para a disponibilização de uma estrutura e de serviços adequados para a população e para o desenvolvimento da capacidade de resposta imediata em situações de emergência (LEE; LEE, 2014). Complementarmente, outros fatores como os investimentos em capital humano e melhorias nas práticas urbanas são condições importantes nesse âmbito (NEIROTTI, et al., 2014). Pode-se afirmar que as smart cities são estruturadas com base em uma combinação promissora de elementos, tais como o capital social, o capital humano, o capital empreendedor e o capital infraestrutural (CARAGLIU; DEL BO; NIJKAMP, 2011; KOURTIT; NIJKAMP, 2012). Assim, as smart cities estão fundamentadas em aspectos e mudanças nas perspectivas tecnológicas e sociais (STÅHLBRÖST et al., 2015), contemplando os seguintes fatores (ZUBIZARRETA; SERAVALLI; ARRIZABALAGA, 2015):

a) economia (competitividade): envolvendo fatores como o espírito inovador, o empreendedorismo, a produtividade, a flexibilidade do mercado de trabalho e a inserção internacional, sendo que esses devem proporcionar um crescimento econômico e social sustentável;

b) pessoas (capital social e humano): ao se considerar que a participação dos cidadãos na vida pública das smart cities é um elemento importante, características como altos níveis de qualificação, pluralidade social e étnica, criatividade e mente aberta, podem ser destacadas;

c) modo de vida (qualidade de vida dos cidadão): essa dimensão está alicerçada na disponibilização de melhores condições de saúde, segurança e educação para os indivíduos. Logo, elementos como as atrações culturais e turísticas, as residências adequadas, a coesão social e a infraestrutura em geral são características relevantes das smart cities;

d) governança (participação dos cidadãos): refere-se às ações governamentais, no sentido de garantir a transparência da utilização de recursos públicos. Além disso, a participação dos cidadãos no pro- cesso de tomada de decisões se estabelece como um aspecto vinculado à dimensão;

e) ambiente (recursos naturais e crescimento sustentável): considerando que o crescimento populacional ocasiona impactos ambientais representativos, a redução do consumo de energia e da poluição, as ações de proteção ambiental e de gestão dos recursos naturais disponíveis são elementos-chave para a sustentabilidade no contexto das smart cities;

f) mobilidade (transportes e tecnologias da informação e comunicação): nas smart cities, fatores como a acessibilidade local e internacional, os sistemas de transportes seguros e sustentáveis e a disponibilidade de infraestrutura em tecnologias da informação e comunicação são aspectos que propiciam a melhoria da mobilidade urbana.

Anttiroiko, Valkama e Bailey (2014) sumarizam esses fatores e afirmam que, embora se verifique que os aspectos tecnológicos representam uma dimensão importante para as smart cities, também é pertinente salientar as perspectivas sociais e ecológicas como essenciais para esse contexto. Para tanto, os autores apontam três dimensões complementares e que se reforçam mutuamente: a dimensão sistêmica, que está vinculada às tecnologias da informação e da comunicação e aos sistemas informatizados e suas funcionalidades; a dimensão social, que representa os interesses dos indivíduos e a qualidade de vida no contexto das smart cities e; a dimensão ambiental, que se relaciona à sustentabilidade dos sistemas ecológicos.

A partir dessas dimensões, é possível inferir a relação existente entre o conceito de smart cities e o desenvolvimento sustentável (BIFULCO et al., 2016). Identifica-se na literatura, inclusive, estudiosos que apresentam o conceito de smart sustainable cities (HÖJER; WANGEL, 2015; AHVENNIEMI et al., 2017), definido como uma cidade que atende às necessidades de seus atuais habitantes, sem comprometer as possibilidades de outros indivíduos ou gerações futuras satisfazem as suas necessidades, sem exceder as limitações locais ou do planeta. Esse processo, de acordo com as premissas apresentadas, é suportado pelas tecnologias da informação e comunicação. Com base nessa visão, a próxima seção 
descreve as principais características conceituais do desenvolvimento sustentável.

\section{DESENVOLVIMENTO SUSTENTÁVEL}

A partir do advento da revolução industrial, a expectativa e a qualidade de vida da população aumentaram. No entanto, consequências como o efeito estufa e a limitação de recursos começaram a ser considerados problemas cruciais, motivando a tomada de consciência para a revisão do atual modelo de desenvolvimento fundamentado no crescimento ilimitado (MEBRATU, 1998; PEREIRA, 2009; SENATORE, 2013). Como uma forma de reação aos desastres oriundos do processo de industrialização, a humanidade formulou uma nova estratégia de desenvolvimento, na qual o meio ambiente era considerado como parte fundamental do processo de evolução da sociedade (PEREIRA, 2009).

Nesse contexto, a partir de 1977, o termo ecodesenvolvimento ganhou ampla atenção, impulsionado por publicações do United Nations Environmental Programme (UNEP), que o via como uma alternativa viável de abranger iniciativas de desenvolvimento que estivessem preocupadas com o meio ambiente, voltando-se à produção do tipo de qualidade de vida desejado, sem destruir a base de recursos da qual o desenvolvimento sustentável depende (UNEP, 1977). Dez anos após a adoção do termo ecodesenvolvimento, a expressão desenvolvimento sustentável passou a ser amplamente difundida e utilizada. Porém, ainda hoje não há um conceito único que a defina (GLAVIČ; LUKMAN, 2007; CLARO; CLARO; AMÂNCIO, 2008; EHNERT, 2009). O que se observa é a compreensão dos recursos a partir de um caráter de perpetuação, envolvendo o crescimento econômico, a preservação ambiental e o bem-estar social (PEREIRA, 2009).

O desenvolvimento sustentável está baseado em um modelo para o gerenciamento do desenvolvimento de comunidades, nações, regiões e do planeta como um todo, objetivando garantir o uso eficiente de recursos, o estabelecimento de infraestruturas eficientes, a proteção e a melhoria da qualidade de vida e a criação de novas empresas para o fortalecimento da economia (PRESLEY; MEADE; SARKIS,
2007). As definições sobre o desenvolvimento sustentável ou sobre a sustentabilidade podem ser as mais variadas e dependem da conjuntura na qual são aplicadas (LEAL FILHO, 2000): o uso sistemático e em longo prazo de recursos naturais, objetivando que os mesmos estejam disponíveis para gerações futuras (contexto de países ou políticas locais); a modalidade de desenvolvimento que permite que países progridam, econômica e socialmente, com a concomitância da preservação dos recursos naturais (referindo-se às políticas de um país); o tipo de desenvolvimento que se apresente como socialmente justo, ético, moral e economicamente sólido (referindo-se às ramificações sociais do desenvolvimento) e; uma tipologia de desenvolvimento na qual os indicadores ambientais são tão importantes quanto os indicadores econômicos (referindo-se aos laços que suportam o desenvolvimento econômico).

Um dos conceitos mais difundidos sobre o desenvolvimento sustentável foi apresentando pela Comissão Mundial para o Meio Ambiente e Desenvolvimento (World Commission on Environment and Development). Para a referida comissão, essa tipologia de desenvolvimento se estabelece como aquela que "satisfaz a necessidade do presente sem comprometer a capacidade de as gerações futuras satisfazerem suas próprias necessidades" (WCED, 1987, p. 54). Logo, a sustentabilidade se apresenta como um princípio que objetiva garantir que as ações atuais não limitem as opções econômicas, sociais e ambientais para as futuras gerações (ELKINGTON, 1997), sendo que essas dimensões podem ser consideradas de forma integrada, com influência entre si (DYLLICK; HOCKERTS, 2002; JOHNSTON et al., 2007). Dyllick e Hockerts (2002) afirmam que a integração de aspectos econômicos, ecológicos e sociais deve ocorrer, considerando que o enfoque exclusivo na sustentabilidade econômica pode proporcionar o sucesso das instituições em curto prazo, enquanto a integração simultânea das três dimensões possibilita uma perspectiva de sustentabilidade em longo prazo.

A difusão das premissas do desenvolvimento sustentável também foi fomentada, inclusive no contexto das instituições, com o surgimento do modelo do triple bottom line (MERRIMAN; SEN, 2012; GLAVAS; MISH, 2015). Esse conceito, de acordo com Elkington (1997), está fundamentado em três pers- 
pectivas: a econômica, a social e a ambiental (profit, people and planet - lucro, pessoas e planeta). $\mathrm{O}$ autor aponta uma interação entre os três elementos, sendo que a sociedade depende de fatores econômicos e a economia, por sua vez, depende de um ecossistema global. Além disso, os elementos componentes do triple bottom line não se apresentam de forma estável e em fluxo constante, em virtude de pressões políticas, econômicas e ambientais, conflitos e mudanças contextuais (ELKINGTON, 1997).

A dimensão econômica da sustentabilidade compreende a economia formal, bem como as atividades informais que promovem serviços para indivíduos e grupos e aumentam a renda monetária e o padrão de vida (CLARO; CLARO; AMÂNCIO, 2008). Além disso, essa perspectiva se relaciona à viabilidade econômica das organizações, ao retorno do investimento realizado e à alocação e ao gerenciamento mais eficiente dos recursos. A eficiência econômica deve ser avaliada em termos macrossociais, visando, além da lucratividade, à promoção de mudanças estruturais que influenciem o desenvolvimento humano e fomentem os investimentos em ciência e tecnologia, em gestão do conhecimento e em inovação (PEREIRA, 2009; BAUMGARTNER; EBNER, 2010).

Em paralelo, destaca-se que a dimensão ambiental da sustentabilidade está centrada no impacto das organizações sobre os ecossistemas e presume que as corporações necessitam ir além do cumprimento de regulamentos governamentais e das ações de reciclagem ou eficiência energética (JAMALI, 2006). A perspectiva ambiental encontra-se em evidência em virtude das mudanças globais, tais como: alterações climáticas e demográficas, urbanização, industrialização, expansão das atividades turísticas e mudança nas formas de uso da terra (WAGNER, 2010). Nesse sentido, a dimensão ambiental está alicerçada na visão do desenvolvimento de sistemas produtivos mais eficientes, com a adoção de práticas ecologicamente corretas e economicamente viáveis, por meio da utilização de tecnologias limpas e fontes renováveis de energia (PEREIRA, 2009; BAUMGARTNER; EBNER, 2010).

A dimensão social visa à igualdade de acesso a bens e serviços de qualidade, requerendo a remoção das principais fontes de desigualdade social e privação da liberdade, tais como: pobreza, tirania, carência de oportunidades econômicas, negligência dos serviços públicos e intolerância ou interferência excessiva dos Estados repressivos (MENDES, 2009). Assim, o desenvolvimento social sustentável está baseado na distribuição equitativa de renda, que possibilita a redução das disparidades dos padrões de vida da população (PEREIRA, 2009). No contexto corporativo, a perspectiva social se relaciona à forma de execução dos processos de produção e desenvolvimento de produtos, afetando os funcionários e a comunidade onde a empresa está inserida (WAAGE et al., 2005). Nesse sentido, essa dimensão contempla questões relacionadas à saúde pública, comunidades, educação, justiça social, segurança e condições de trabalho, direitos humanos, igualdade de oportunidades e direitos trabalhistas (JAMALI, 2006).

No âmbito organizacional, o triple bottom line visa analisar a performance dos negócios e seus impactos na lucratividade, nos stakeholders e no aspecto ambiental (DAO; LANGELLA; CARBO, 2011). A partir dessa abordagem, além do objetivo de desempenho econômico, as instituições precisam se envolver em atividades que afetam, de maneira positiva, o meio ambiente e a sociedade. Assim, essa concepção está embasada em três princípios: a integridade ambiental, a prosperidade econômica e a equidade social (HAHN; FIGGE, 2011). Perrini e Tencati (2006) corroboram com essa visão e afirmam que uma organização orientada para a sustentabilidade é aquela que se desenvolve ao longo do tempo, considerando as dimensões econômica, social e ambiental em seus processos e em relação ao seu desempenho. Nessa perspectiva, o sucesso financeiro e competitivo, a legitimidade social e o uso eficiente de recursos naturais, devem estar interligados de acordo com uma visão sinérgica dos objetivos da empresa.

\section{PROCEDIMENTOS METODOLÓGICOS}

Conforme mencionado anteriormente, o presente artigo tem como objetivo caracterizar os estudos que vinculam os temas smart cities e desenvolvimento sustentável, além de identificar oportunidades de pesquisas futuras nesse contexto. Para tanto, optou-se por realizar uma revisão sistemática de literatura, que contempla a investigação científica e inclui uma pes- 
quisa abrangente de artigos potencialmente relevantes e a utilização de critérios explícitos e reprodutíveis de seleção. Logo, a revisão sistemática possibilita reunir, avaliar e sintetizar resultados de múltiplos estudos (COOK, MULROW E HAYNES, 1997). Wolfswinkel, Furtmueller e Wilderom (2013) sugerem o suporte do método da Teoria Fundamentada para a revisão sistemática de literatura, através de cinco estágios: definir os critérios de inclusão e exclusão de artigos, campos e disciplinas de pesquisa, bases de dados e termos específicos a serem consultados; realizar a pes- quisa efetiva, através das fontes identificadas; realizar a seleção dos artigos e refinar a amostra; analisar os artigos e desenvolver o processo de codificação e/ou classificação e; estruturar e apresentar os resultados.

Com o objetivo de evidenciar os principais enfoques teóricos sobre os estudos que abordam os temas smart cities e desenvolvimento sustentável, são apresentados, na Figura 1, os critérios utilizados para a elaboração da revisão sistemática, de acordo as fases propostas por Wolfswinkel, Furtmueller e Wilderom (2013).

Figura 1 Critérios para a revisão sistemática de literatura

\begin{tabular}{|c|c|}
\hline Estágio & Critérios e ações realizadas \\
\hline Definir & $\begin{array}{l}\text { Pesquisa realizada na base de dados Scopus. } \\
\text { Seleção de artigos publicados em periódicos científicos. } \\
\text { Busca de estudos que apresentassem os seguintes termos nas palavras-chave: } \\
\text { Smart cit* and Sustainable Development }\end{array}$ \\
\hline \multicolumn{2}{|r|}{ 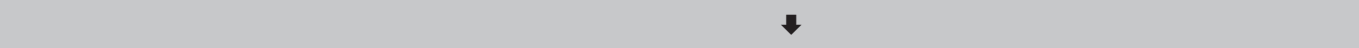 } \\
\hline Pesquisar & - Realização da pesquisa entre julho e outubro de 2016. \\
\hline \multicolumn{2}{|r|}{ 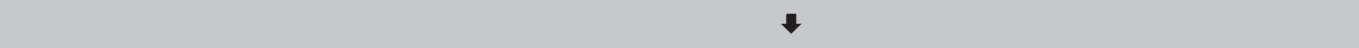 } \\
\hline Selecionar & - Seleção de documentos em formato de artigo científico, apresentados nos idiomas inglês ou português. \\
\hline \multicolumn{2}{|r|}{$\sqrt{-1}$} \\
\hline Analisar & - Análise dos artigos selecionados com o suporte do software Alceste. \\
\hline \multicolumn{2}{|r|}{$\sqrt{n}$} \\
\hline $\begin{array}{l}\text { Apresen- } \\
\text { tar }\end{array}$ & - Representação e discussão dos resultados da pesquisa, descritos na Seção 5 do presente estudo. \\
\hline
\end{tabular}

Fonte: Elaborada pelos autores, a partir das sugestões de Wolfswinkel, Furtmueller e Wilderom (2013).

Também considera-se importante apresentar alguns aspectos e critérios comuns que foram observados para o desenvolvimento da revisão sistemática do presente estudo. Optou-se pela realização das buscas na base de dados Scopus, levando-se em consideração que a mesma se apresenta como a maior base de resumos e citações de literatura científica, revisada por pares. Além disso, a base de dados Scopus disponibiliza ferramentas para acompanhar, analisar e visualizar as pesquisas realizadas em revistas científicas, livros e anais de eventos (LEYDESDORFF; MOYA-ANEGÓN; GUERRERO-BOTE, 2015; SCOPUS, 2016). Também limitou-se a pesquisa por artigos publicados nos idiomas português ou inglês e disponíveis em periódicos científicos. Nesse caso, não foram considerados os artigos apresentados em congressos ou outros eventos, uma vez que esses são considerados estudos em construção. Por fim, não ocorreu nenhuma restrição em termos de período de publicação dos estudos.

A partir da definição dos critérios apresentados na Figura 1 e da realização da pesquisa em si, foram identificados, inicialmente, 577 artigos que apresentavam o termo "smart cit" (cidades inteligentes) e 42.926 estudos com a expressão "sustainable development" (desenvolvimento sustentável) em suas palavras-chave. Com base na junção desses critérios, evidenciou-se 34 estudos que abordavam o tema smart cities e desenvolvimento sustentável, de forma concomitante. Desses, durante o processo de seleção, 
optou-se por desconsiderar sete estudos. Para tanto, observou-se que dois documentos estavam em formato de editorial, não se enquadrando no critério de analisar apenas artigos científicos. Além disso, cinco estudos não obedeciam aos critérios em relação ao idioma, não estando redigidos em português ou inglês. Por fim, 27 artigos foram selecionados e submetidos à fase de análise.

Após a realização do processo de refinamento mencionado, realizou-se a leitura dos resumos e, posteriormente, do conteúdo geral dos artigos, objetivando identificar as principais características metodológicas, considerando se os estudos apresentavam cunho teórico ou empírico e quantitativo, qualitativo ou misto. Além disso, foram observadas as principais estratégias de pesquisa e de análise de dados nos estudos selecionados.

Na sequência da realização dessas análises, os resumos das publicações selecionadas foram organizados em arquivo específico, objetivando a posterior análise do conteúdo, com apoio do software Alceste (Análise Lexical Contextual de um Conjunto de Segmentos de Texto), versão 2015. Uma das principais vantagens da utilização desse programa é o processamento automático de um volume amplo de dados, com a interferência de um único pesquisador. Esse fato reduz a subjetividade que ocorre, frequentemente, durante a aplicação de análises de conteúdo tradicionais e na execução de codificações realizadas manualmente por um pesquisador (COLUCCI; MONTALI, 2008). Esse programa permite realizar, de forma automática, a análise de entrevistas, obras literárias, artigos, ensaios e outros formatos de texto, com o propósito de quantificar e extrair suas estruturas mais significativas, através da aplicação do método de classificação hierárquica descendente (CAMARGO, 2005; ILLIA; SONPAR; BAUER, 2014).

Nesses processamentos, ocorre a subdivisão de unidades de contexto em classes que apresentam vocabulário semelhante entre si, porém diferente das unidades de contexto das demais classes (ABITAN; KRAUTH-GRUBER, 2015). Para tanto, o software utiliza o teste do qui-quadrado para verificar a associação das unidades de contexto às respectivas classes (CAMARGO, 2005). As denominações das classes que emergem a partir da análise do software Alceste podem ser comparadas às definições utilizadas para indicar as dimensões latentes resultantes de uma análise fatorial em pesquisas quantitativas. Além disso, se as definições apresentarem um significado reconhecido e suportado pela literatura, há indicação de que a análise realizada é significativa e válida (COLUCCI; MONTALI, 2008).

Durante o processo de análise dos estudos, com o suporte do software Alceste, definiu-se que o conjunto textual a ser explorado (corpus) seria composto pelos resumos dos artigos selecionados na terceira etapa da revisão sistemática, totalizando 27 unidades de contexto inicial (UCIs). As UCIs foram processadas, originando 136 unidades de contexto elementar (UCEs), que representam segmentos do texto, geralmente com três linhas, dimensionadas pelo programa em função do tamanho do corpus e, em geral, respeitando a pontuação (CAMARGO, 2005). Como principais resultados, foram identificadas duas classes que contemplaram $48 \%$ do total das UCEs do corpus analisado. As demais análises desse processo estão descritas na próxima seção do estudo.

\section{ANÁLISES E DISCUSSÃO}

Inicialmente, buscou-se identificar as principais características dos estudos que abordavam o tema smart cities ou desenvolvimento sustentável. Nesse sentido, identificou-se a existência de 577 artigos que apresentavam o tema smart cities nas palavras-chave. Também verificou-se que a maior parte das pesquisas foi desenvolvida entre os anos de 2014 e 2016, representando 83\% dos estudos identificados nessa verificação preliminar. O Gráfico 1 apresenta a evolução do número de publicações que abordam o assunto smart cities, no qual é possível destacar a tendência de crescimento de pesquisas sobre o tema. Ao mesmo tempo, confirma-se a contemporaneidade dos estudos, tendo em vista que, na base de dados Scopus, localizou-se artigos publicados somente a partir do ano de 2009. Além disso, detectou-se uma predominância de estudos originados nos seguintes países: Itália (16\%), Espanha (16\%), China (12\%), Estados Unidos (12\%), Reino Unido (11\%) e Grécia (6\%). Esse resultado destaca alguns países europeus no desenvolvimento de pesquisas sobre smart cities. 
Gráfico 1 Número de publicações anuais sobre o tema smart cities

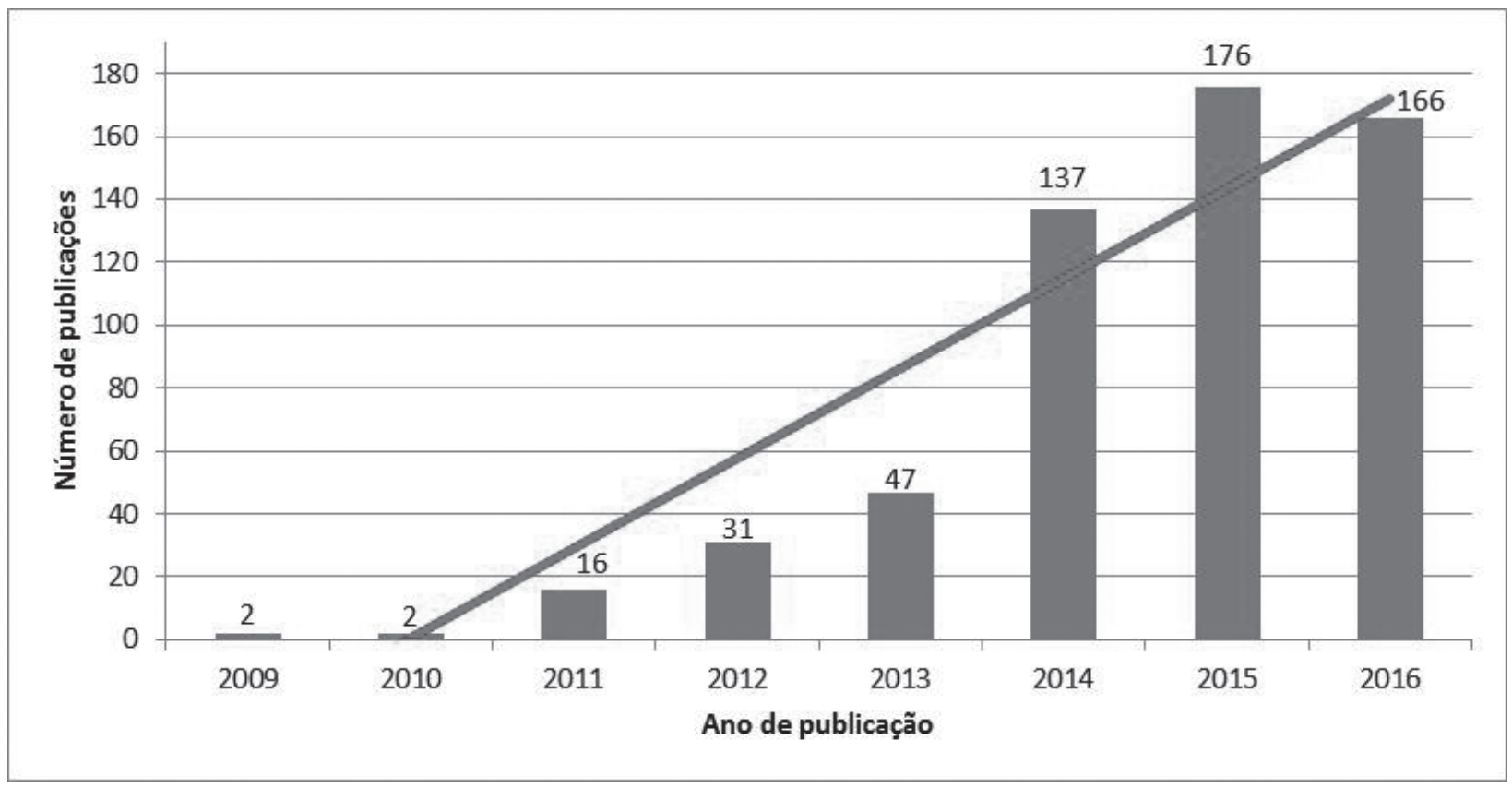

Fonte: Elaborado pelos autores, com base nos resultados da pesquisa.

Na sequência, realizou-se a verificação dos estudos que tratam sobre o tema desenvolvimento sustentável, publicados a partir de 2009, considerando que após esse período foram desenvolvidos os estudos sobre smart cities. Com base nos mesmos critérios observados na revisão sistemática sobre smart cities, foram identificados 26.016 artigos. Nesse sentido, embora se destaque um número representativo de publicações, com base em três décadas de pesquisas, ainda percebe-se a importância da realização de estudos sobre desenvolvimento sustentável, ao se considerar o tendência de crescimento das pesquisas, conforme apontado no Gráfico 2. Também analisou-se os principais países de origem das publicações, com destaque para Estados Unidos (19\%), Reino Unido (11\%), China (11\%), Austrália (6\%), Itália (5\%), Alemanha (5\%) e Canadá (5\%).

Posteriormente, focando o objetivo principal do presente estudo, realizou-se a avaliação dos 27 artigos que abordavam o tema smart cities e desenvolvimento sustentável, de forma concomitante. Em relação ao período de publicação, observou-se que $63 \%$ dos estudos foram publicados entre os anos de 2014 e 2015. Os estudos também foram analisados, inicialmente, de acordo com alguns aspectos metodológicos. Nesse sentido, verificou-se que $67 \%$ dos artigos são de cunho empírico e 33\% de cunho teórico. Identificou-se uma predominância de estudos qualitativos (63\%), com menor incidência de pesquisas quantitativas (19\%) e mistas (18\%). Em relação às estratégias de pesquisa, constatou-se que as revisões de literatura (48\%) e os estudos de caso (37\%) foram as abordagens prevalecentes. Por fim, 59\% dos artigos avaliados empregaram técnicas de análise de conteúdo para a avaliação dos resultados ou promoção das discussões. Além disso, 37\% dos estudos utilizaram alguma técnica de análise estatística para suportar os resultados identificados. 
Gráfico 2 Número de publicações anuais sobre o tema desenvolvimento sustentável

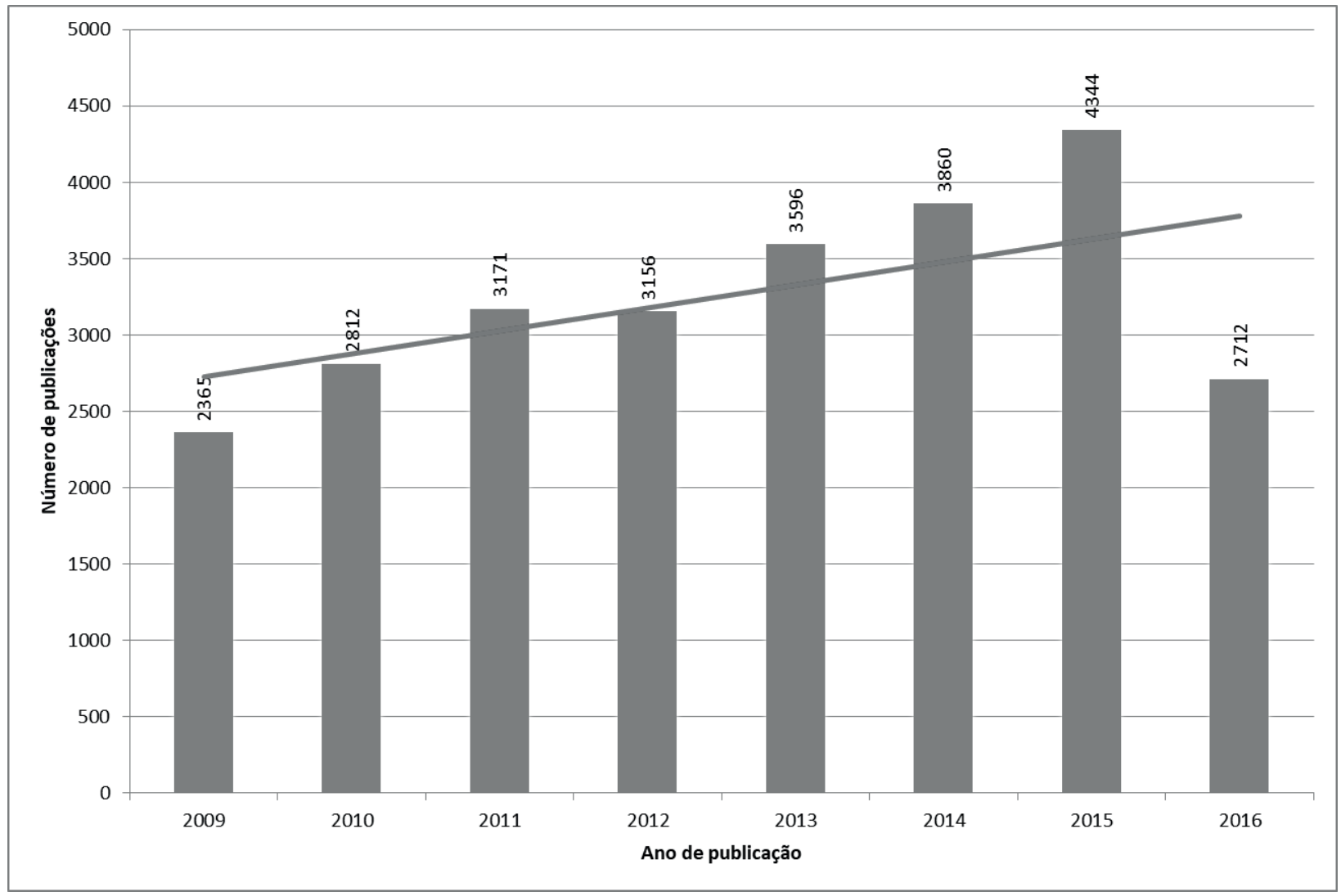

Fonte: Elaborado pelos autores, com base nos resultados da pesquisa.

Com o objetivo de constatar as principais vertentes dos estudos que abordam o desenvolvimento sustentável no contexto das smart cities, realizou-se a análise dos resumos dos artigos, com o apoio do software Alceste. A partir dos resultados iniciais, verificou-se a existência de duas categorias distintas, que emergiram da avaliação de 136 unidades de contexto elementar (UCEs). Destaca-se que as classes identificadas contemplaram $48 \%$ do total das UCEs do corpus analisado. Na sequência, examinou-se os termos mais expressivos que compuseram cada categoria, de acordo com os seguintes critérios de seleção: evidenciou-se os termos não instrumentais com média de frequência superior a 3 (média calculada pelo software Alceste) e; considerou-se as palavras associadas à classe com o valor do qui-quadrado igual ou superior a 3,84, uma vez que o cálculo da associação é fundamentado em um teste estatístico com base em uma tabela com grau de liberdade igual a 1 (CAMARGO, 2005). Os resultados dessa análise estão representados no dendograma exposto na Figura 2.
Figura 2 Dendograma e classes identificadas

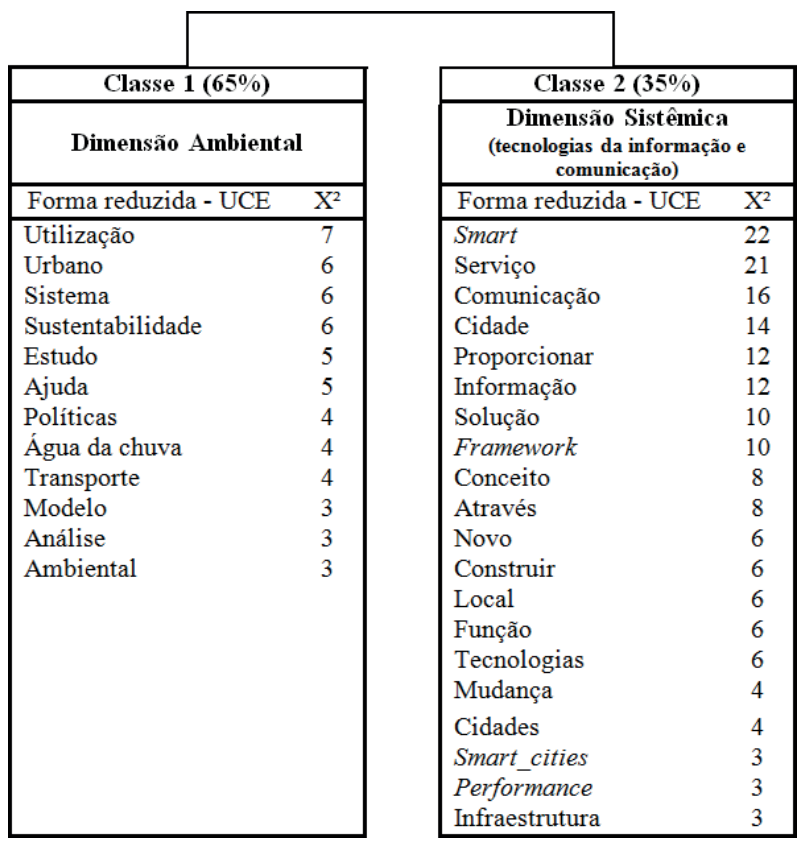

Fonte: Elaborada pelos autores, a partir dos resultados processados pelo software Alceste. 
A categoria mais significativa, denominada de Dimensão Ambiental, representa 65\% das UCEs. Os estudos que compõem essa classe estão alicerçados na ideia de que as áreas urbanas são as maiores consumidoras de recursos naturais cada vez mais escassos, sendo que, desse contexto, emerge a necessidade de tornar as cidades mais sustentáveis no que se refere à perspectiva ambiental (FERNÁNDEZ et al., 2014; ZUBIZARRETA; SERAVALLI; ARRIZABALAGA, 2015). Nessa mesma linha, destaca-se que o desenvolvimento e a riqueza das cidades, em muitas situações, dependem dos recursos naturais e das atividades turísticas, sendo que a exploração desses elementos deve ocorrer de maneira segura e responsável (CARAGLIU; DEL BO; NIJKAMP, 2011).

A partir desse cenário, a utilização adequada das tecnologias deve proporcionar o melhor gerenciamento dos recursos naturais e promover a sustentabilidade ambiental e a viabilidade ecológica da cidade (CHOURABI et al., 2012). Como exemplo, pode-se citar: a aplicação das tecnologias para a redução do consumo de recursos, como água e energia; o maior aproveitamento da infraestrutura existente, reduzindo a necessidade de novos projetos ou construções; a disponibilização de serviços aos cidadãos, para que as diversas modalidades de transporte sejam utilizadas de maneira mais eficiente e; o monitoramento das necessidades de energia, água e transportes, que possibilitará que as organizações gerenciadoras destes recursos ou serviços atuem com maior resiliência em relação às demandas (HARRISON; DONNELLY, 2011).

Já a segunda categoria, que contempla $35 \%$ das UCEs identificadas, foi nomeada como Dimensão Sistêmica, por contemplar alguns elementos vinculados às tecnologias da informação e comunicação, destacados por Anttiroiko, Valkama e Bailey (2014). Essa classe abrange os estudos que enfocam a caracterização das smart cities a partir de aspectos como a utilização eficiente de recursos e a aplicação de tecnologias da informação e comunicação (CORSINI; RIZZI; FREY, 2016), que viabilizaram uma vasta gama de soluções e produtos vinculados a esse âmbito (ANGELIDOU, 2015).

As tecnologias de informação e comunicação desempenham um papel crucial nas smart cities, sendo consideradas componentes essenciais para o desen- volvimento de iniciativas nesse contexto (SÁNCHEZ et al., 2003; CARAGLIU; DEL BO; NIJKAMP, 2011; ALAWADHI et al., 2012; PIRO et al., 2014; MARCH, 2016). Essas tecnologias auxiliam no aproveitamento eficiente da infraestrutura disponível, na construção de redes de comunicação e no fomento dos processos que tornam os cidadãos mais bem informados. Nesse sentido, a integração entre as tecnologias e o âmbito das smart cities estimula a prosperidade e o crescimento econômico (SÁNCHEZ et al., 2003).

Em convergência, é possível destacar que, no ambiente das smart cities, o desempenho urbano pode ser alavancado através da utilização de dados, informações e tecnologias (TICs), com o objetivo de fornecer serviços mais eficientes aos cidadãos, com base na monitoração e utilização eficaz da infraestrutura existente (HARRISON, DONNELLY, 2011). Essa situação possibilita a colaboração entre os agentes econômicos e o desenvolvimento de modelos inovadores de negócios nos setores público e privado (MARSAL-LLACUNA; COLOMER-LLINÃS; MELÉNDEZ-FRIGOLA, 2015). Em suma, a utilização adequada das tecnologias da informação e comunicação proporciona a melhoria dos serviços prestados e a consequente ampliação da qualidade de vida dos cidadãos, sendo que esse cenário favorece o desenvolvimento sustentável (BIFULCO et al., 2016).

A partir dos resultados da revisão sistemática de literatura, bem como dos enfoques dos artigos analisados, é possível reforçar que, no contexto das smart cities, identifica-se a integração das dimensões do desenvolvimento sustentável. Destaca-se que essa tipologia de cidade combina seus recursos, de maneira inteligente, para proporcionar melhores condições econômicas, ambientais e sociais para a população (ALAWADHI et al., 2012). Cassandras (2016) corrobora com essas perspectivas, ao afirmar que as smart cities desenvolvem um ambiente urbano alicerçado na disponibilização de serviços inovadores, na existência de negócios competitivos, na distribuição adequada de energia, no monitoramento ambiental, nos cuidados com a saúde da população e na promoção de atividades sociais.

Nesse sentido, as tecnologias da informação e comunicação apresentam-se como ferramentas que favorecem o monitoramento, a análise e a otimização (KUTAMI; TAKENO; IOKA, 2014) dos fluxos de 
recursos e bens (dimensão econômica), dos fatores ambientais (dimensão ambiental) e dos aspectos vinculados às pessoas e suas relações (dimensão social). Logo, ocorre nas smart cities a adoção intensiva de tecnologias, que possibilitam a conexão entre informações, pessoas e outros elementos da cidade, com o objetivo de desenvolver um ambiente sustentável, com uma economia inovadora e competitiva e com qualidade de vida para seus cidadãos (BAKICI; ALMIRALL; WAREHAM, 2013).

No entanto, segundo o processamento dos dados, com o suporte do software Alceste, identificou-se a existência de dois enfoques predominantes nos estudos vinculados ao contexto das smart cities: ênfase na sustentabilidade ambiental e no desenvolvimento e aplicação das tecnologias da informação e comunicação. Em contrapartida, vislumbra-se a oportunidade de desenvolvimento de estudos futuros que contemplem a dimensão social e econômica da sustentabilidade, incluindo a criação de indicadores que mensurem esses aspectos (AHVENNIEMI et al., 2017). A Figura 3 sumariza as perspectivas identificadas na revisão sistemática de literatura.

Figura 3 Perspectivas identificadas na revisão sistemática de literatura

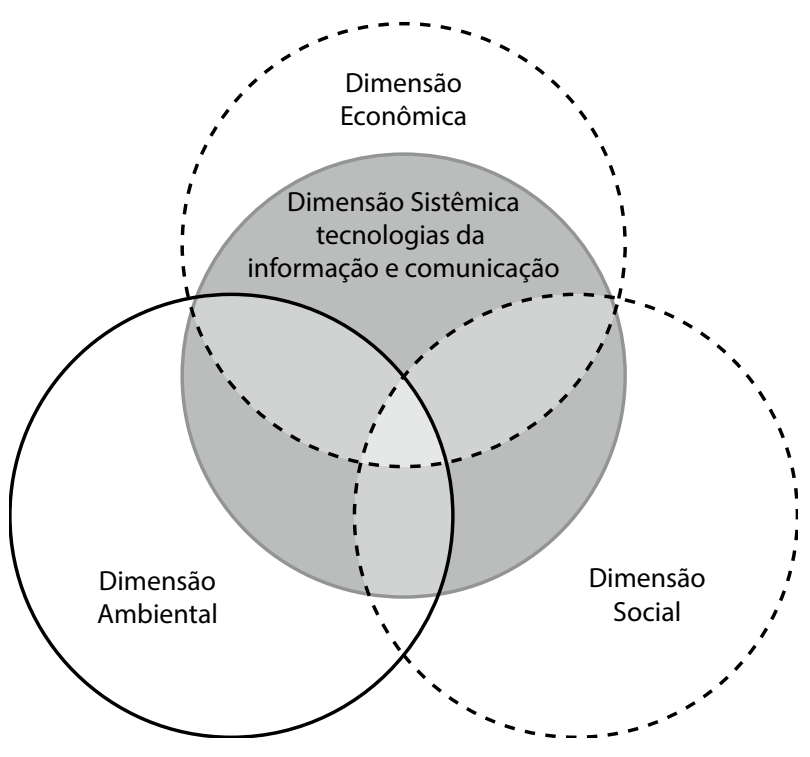

Fonte: Elaborada pelos autores, a partir dos resultados da revisão sistemática de literatura.

Com base no conteúdo apresentado na Figura 3, verifica-se que as tecnologias da informação e comunicação se apresentam como um elemento relevante para o desenvolvimento sustentável das smart cities. Além disso, destaca-se, as dimensões da sustentabilidade (econômica e social) que podem ser mais exploradas a partir do desenvolvimento de estudos futuros.

Essa constatação também está embasada em alguns apontamentos da literatura. Nesse sentido, percebe-se que, em muitas situações, o conceito de smart cities é vinculado apenas aos aspectos tecnológicos (CORSINI; RIZZI; FREY, 2016). Todavia, identifica-se a necessidade de uma abordagem mais holística, integrada e multidisciplinar (ALBINO; BERARDI; DANGELICO, 2015; ZUBIZARRETA; SERAVALLI; ARRIZABALAGA, 2015), contemplando também os aspectos sociais e ambientais relacionados ao desenvolvimento sustentável (ANTTIROIKO; VALKAMA; BAILEY, 2014).

\section{CONSIDERAÇÕES FINAIS}

O desenvolvimento sustentável apresenta-se como uma dimensão que depende de fatores que ultrapassam as variáveis econômicas. Com base nesta premissa, as smart cities, através da aplicação do conhecimento intensivo e de estratégias criativas e inovadoras, enfocam a melhoria do desempenho social e ambiental e a expansão da economia e da competitividade das cidades (KOURTIT; NIJKAMP, 2012). Nesse contexto, partindo da integração entre os temas smart cities e desenvolvimento sustentável, o presente estudo contempla algumas contribuições consideradas relevantes para esse campo de pesquisa.

Primeiramente, a partir dos artigos analisados, identificou-se a predominância de estudos qualitativos, em detrimento das abordagens quantitativas ou mistas. Esse resultado é condizente, ao se considerar que as pesquisas sobre smart cities são recentes. Com base nesse resultado, sugere-se o desenvolvimento de estudos futuros que adotem a perspectiva quantitativa de pesquisa. Essa possibilidade poderá, inclusive, colmatar a lacuna de pesquisa que se relaciona à avaliação das smart cities. Além disso, embora a literatura aponte a integração entre os temas smart cities e sustentabilidade, verifica-se que ainda é possível salientar desafios que se originam desse contexto: o desenvolvimento e aplicação de métodos que auxiliem a avaliar 
se uma smart city é, de fato, sustentável; a criação de estratégias para o fortalecimento das competências governamentais e o aperfeiçoamento de modelos de governança que propiciem o desenvolvimento sustentável das smart cities (LEE; HANCOCK; HU, 2014; HÖJER; WANGEL, 2015).

O presente estudo também contribuiu com direcionamentos futuros no que se refere às dimensões da sustentabilidade a serem mais exploradas no contexto das smart cities. Os resultados da revisão sistemática de literatura, suportada pelo processamento dos dados pelo software Alceste, apontaram que os artigos que integram as temáticas centrais desse estudo enfocam, predominantemente, a dimensão ambiental da sustentabilidade e a utilização das tecnologias de informação e comunicação (denominada como dimensão sistêmica, conforme disposto por Anttiroiko, Valkama e Bailey (2014)) no contexto das smart cities. A partir desses resultados, identifica-se a oportunidade de desenvolvimento de estudos que considerem as dimensões econômica e social da sustentabilidade.

Em relação à dimensão econômica, identificou-se, a partir da revisão sistemática de literatura, a oportunidade de realização de estudos futuros que explorem: as relações entre a capacidade de atração de investimentos privados e atributos das smart cities (CORSINI; RIZZI; FREY, 2016) e; a criação de políticas públicas que auxiliem na criação e difusão de inovações sustentáveis e apoiem o desenvolvimento econômico local (COHEN; AMORÓS, 2014).

No tocante à dimensão social, aponta-se a possibilidade de realização de estudos que analisem o papel do capital social e do capital relacional no desenvolvimento das smart cities, além de verificar os efeitos da polarização social, nas esferas econômica, geográfica e cultural (CARAGLIU; DEL BO; NIJKAMP, 2011). Além disso, pesquisas futuras podem enfocar, com maior profundidade, a correlação existente entre o contexto das smart cities e o desempenho sustentável (AHVENNIEMI et al., 2017).

Também se considera relevante indicar algumas limitações do estudo, que poderão, inclusive, fomentar o desenvolvimento de futuras pesquisas. Inicialmente, uma das limitações do estudo foi a não inclusão de artigos apresentados em congressos ou outros eventos, no processo de revisão sistemática de literatura. Essa decisão foi baseada no fato de que es- ses estudos são considerados trabalhos em construção. No entanto, artigos apresentados em eventos poderão trazer novas abordagens sobre os temas smart cities e desenvolvimento sustentável. Sendo assim, sugere-se que estudos futuros incluam essa tipologia de artigo, para posterior comparação com os resultados do presente estudo.

Outra possível limitação da pesquisa está relacionada à análise do tema smart cities com uma perspectiva genérica do desenvolvimento sustentável. Nesse caso, novas revisões de literatura poderiam enfocar, de maneira mais específica, o vínculo do tema smart cities com a sustentabilidade econômica, a sustentabilidade social e a sustentabilidade ambiental.

Com base na argumentação apresentada, destaca-se que o presente estudo não pretende esgotar a discussão sobre os resultados encontrados. Em contrapartida, acredita-se que esta pesquisa possa contribuir para a avaliação de temas emergentes de pesquisa sobre smart cities e desenvolvimento sustentável, bem como embasar estudos futuros.

\section{AGRADECIMENTOS}

Este trabalho está sendo apoiado pelas seguintes instituições: Universidade de Caxias do Sul (UCS), IMED Business School (IMED), Instituto Federal de Educação, Ciência e Tecnologia do Rio Grande do Sul (IFRS), Coordenação de Aperfeiçoamento do Pessoal de Nível Superior (CAPES) e Conselho Nacional de Desenvolvimento Científico e Tecnológico (CNPq).

\section{REFERÊNCIAS}

ABITAN, A.; KRAUTH-GRUBER, S. The two sides of disgust: A lexical and thematic content analysis of narratives of personally experienced physical and moral disgust. Social Science Information, v. 54, n. 4 , p. 470-496, 2015.

AHVENNIEMI, Hannele et al. What are the differences between sustainable and smart cities?. Cities, v. 60, p. 234-245, 2017. 
ALAWADHI, S. et al. Building understanding of smart city initiatives. In: SCHOLL, H. J.; WIMMER, M.; MOE, C.; FLAK, L. (Eds.). Electronic Government. Springer Berlin / Heidelberg. v. 7443, 2012, p. 40-53.

ALBINO, V.; BERARDI, U.; DANGELICO, R. M. Smart cities: definitions, dimensions, performance, and initiatives. Journal of Urban Technology, v. 22, n. 1, p. 3-21, 2015.

ANGELIDOU, M. Smart cities: A conjuncture of four forces. Cities, v. 47, p. 95-106, 2015.

ANTTIROIKO, A.; VALKAMA, P.; BAILEY, S. J. Smart cities in the new service economy: building platforms for smart services. AI \& Society, v. 29, n. 3, p. 323-334, 2014.

BAKICI, T.; ALMIRALL, E.; WAREHAM, J. A smart city initiative: the case of Barcelona. Journal of the Knowledge Economy, v. 4, n. 2, p. 135-148, 2013.

BATTY, M. et al. Smart cities of the future. The European Physical Journal Special Topics, v. 214, n. 1, p. 481-518, 2012.

BAUMGARTNER, R. J.; EBNER, D. Corporate sustainability strategies: sustainability profiles and maturity levels. Sustainable Development, v. 18, n. 2, p. 76-89, 2010.

BIFULCO, F. et al. ICT and sustainability in smart cities management. International Journal of Public Sector Management, v. 29, n. 2, p. 132-147, 2016.

BULLER, P. F.; MCEVOY, G. M. A model for implementing a sustainability strategy through HRM practices. Business and Society Review, v. 121, n. 4, p. $465-495,2016$.

CAMARGO, B. V. ALCESTE: um programa informático de análise quantitativa de dados textuais. In: MOREIRA, Antonia Silva Paredes et al. Perspectivas teórico-metodológicas em representações sociais, João Pessoa: Editora Universitária UFPB, 2005, p. 511-539.
CARAGLIU, A.; DEL BO, C.; NIJKAMP, P. Smart cities in Europe. Journal of Urban Technology, v. 18, n. 2, p. 65-82, 2011.

CARAGLIU, A. et al. Smart Cities. International Encyclopedia of the Social \& Behavioral Sciences, v. 22, p. 113-117, 2015.

CASSANDRAS, C. G. Smart Cities as Cyber-Physical Social Systems. Engineering, v. 2, n. 2, p. 156-158, 2016.

CHOURABI, H. et al. Understanding smart cities: an integrative framework. In: System Science (HICSS), 45th Hawaii International Conference on. IEEE, p. 2289-2297, 2012.

ClARO, P. B. O.; ClARO, D. P.; AMÂNCIO, R. Entendendo o conceito de sustentabilidade nas organizações. Revista de Administração, v. 43, n. 4, p. 289-300, 2008.

COHEN, B.; AMORÓS, J. E. Municipal demand-side policy tools and the strategic management of technology life cycles. Technovation, v. 34, n. 12, p. 797 806, 2014.

COLUCCI, Francesco Paolo; MONTALI, Lorenzo. Comparative application of two methodological approaches to the analysis of discourses. International Journal of Multiple Research Approaches, v. 2, n. 1, p. 57-70, 2008.

COOK, D. J.; MULROW, C. D.; HAYNES, R. B. Systematic reviews: synthesis of best evidence for clinical decisions. Annals of Internal Medicine. v. 126, n. 5, p. 376-380, 1997.

CORSINI, F.; RIZZI, F.; FREY, M. Analysing smartness in European cities: a factor analysis based on resource efficiency, transportation and ICT. International Journal of Global Environmental Issues, v. 15, n. 3, p. 235-254, 2016. 
DAO, V.; LANGELLA, I.; CARBO, J. From green to sustainability: Information Technology and an integrated sustainability framework. The Journal of Strategic Information Systems, v. 20, n. 1, p. 63-79, 2011.

DATTA, A. New urban utopias of postcolonial India 'Entrepreneurial urbanization'in Dholera smart city, Gujarat. Dialogues in Human Geography, v. 5, n. 1, p. 3-22, 2015.

DYLLICK, T.; HOCKERTS, K. Beyond the business case for corporate sustainability. Business Strategy and the Environment, v. 11, n. 2, p. 130-141, 2002.

DYLLICK, T.; MUFF, K. Clarifying the Meaning of Sustainable Business: Introducing a Typology From Business-as-Usual to True Business Sustainability. Organization \& Environment, v. 1, n. 19, p. 1-19, 2015.

ELKINGTON, J. Cannibals with forks: the triple bottom line of 21st century, United Kingdom: Capstone Publishing, 1997.

EHNERT, I. Sustainable Human Resource Management: A conceptual and explanatory analysis from a paradox perspective. Contributions to management science. Heidelberg: Springer-Verlag, 2009.

FERNÁNDEZ, C. et al. Modeling energy consumption in automated vacuum waste collection systems. Environmental Modelling \& Software, v. 56, p. 6373, 2014.

GIFFINGER, R. et al. Smart cities: Ranking of European medium-sized cities (Report). Vienna University of Technology, 2007.

GLAVAS, A.; MISH, J. Resources and Capabilities of Triple Bottom Line Firms: Going Over Old or Breaking New Ground?. Journal of Business Ethics, v. 127, n. 3, p. 623-642, 2015.

GLAVIČ, P.; LUKMAN, R. Review of sustainability terms and their definitions. Journal of Cleaner Production, v. 15, n. 18, p. 1875-1885, 2007.
GUERCI, M.; SHANI, A. B. R.; LUCA, S. A Stakeholder Perspective for Sustainable HRM: Literature Review and a Research Agenda. In: EHNERT, I.; HARRY, W.; ZINK, K. J. (Ed.). Sustainability and human resource management: Developing sustainable business organizations. Germany: Springer Science \& Business Media, 2013, p. 205-223.

HAHN, T.; FIGGE, F. Beyond the bounded instrumentality in current corporate sustainability research: Toward an inclusive notion of profitability. Journal of Business Ethics, v. 104, n. 3, p. 325-345, 2011.

HARRISON, C.; DONNELLY, I., A. A theory of smart cities. Proceedings of the 55th Annual Meeting of the International Society for the Systems Sciences. p. 2-15, 2011.

HÖJER, M.; WANGEL, J. Smart sustainable cities: definition and challenges. ICT Innovations for Sustainability. Springer International Publishing, v. 310, p. 333-349, 2015.

HOLLANDS, R. G. Will the real smart city please stand up? Intelligent, progressive or entrepreneurial?. City, v. 12, n. 3, p. 303-320, 2008.

ILLIA, L.; SONPAR, K.; BAUER, M. W. Applying Co-occurrence Text Analysis with ALCESTE to Studies of Impression Management. British Journal of Management, v. 25, n. 2, p. 352-372, 2014.

JAMALI, D. Insights into triple bottom line integration from a learning organization perspective. Business Process Management Journal, v. 12, n. 6, p. 809-821, 2006.

JAMALI, D. A stakeholder approach to corporate social responsibility: A fresh perspective into theory and practice. Journal of Business Ethics, v. 82, n. 1, p. 213-231, 2008.

JOHNSTON, P. et al. Reclaiming the definition of sustainability. Environmental Science and Pollution Research, v. 14, n. 1, p. 60-66, 2007. 
KOURTIT, K.; NIJKAMP, P. Smart cities in the innovation age. Innovation: The European Journal of Social Science Research, v. 25, n. 2, p. 93-95, 2012.

KUTAMI, M.; TAKENO, M.; IOKA, H.. New Approach for Environmental Future City Created by ICT: Sustainable City Network. Fujitsu Scientific \& Technical Journal, v. 50, n. 2, p. 100-111, 2014.

LAZAROIU, G. C.; ROSCIA, M. Definition methodology for the smart cities model. Energy, v. 47, n. 1, p. 326-332, 2012.

LEAL FILHO, W. Dealing with misconceptions on the concept of sustainability. International Journal of Sustainability in Higher Education, v. 1, n. 1, p. 9-19, 2000.

LEAL FILHO, W. et al. Reinvigorating the sustainable development research agenda: the role of the sustainable development goals (SDG). International Journal of Sustainable Development \& World Ecology, v. 25, n. 2, p. 131-142, 2018.

LEE, J.; HANCOCK, M. G.; HU, M.. Towards an effective framework for building smart cities: Lessons from Seoul and San Francisco. Technological Forecasting and Social Change, v. 89, p. 80-99, 2014.

LEE, J.; LEE, H. Developing and validating a citizen-centric typology for smart city services. Government Information Quarterly, v. 31, p. 93-105, 2014.

LEYDESDORFF, Loet; MOYA-ANEGÓN, Félix; GUERRERO-BOTE, Vicente P. Journal maps, interactive overlays, and the measurement of interdisciplinarity on the basis of Scopus data (1996-2012). Journal of the Association for Information Science and Technology, v. 66, n. 5, p. 1001-1016, 2015.

LINNENLUECKE, M. K.; GRIFFITHS, A. Corporate sustainability and organizational culture. Journal of World Business, v. 45, n. 4, p. 357-366, 2010.
MARCH, H. The Smart City and other ICT-led techno-imaginaries: Any room for dialogue with Degrowth?. Journal of Cleaner Production, article in press, p. 1-10, 2016.

MARSAL-LLACUNA, M.; COLOMER-LLINÀS, J.; MELÉNDEZ-FRIGOLA, J. Lessons in urban monitoring taken from sustainable and livable cities to better address the Smart Cities initiative. Technological Forecasting and Social Change, v. 90, p. 611-622, 2015.

MASSANA, J. et al. Identifying services for shortterm load forecasting using data driven models in a Smart City platform. Sustainable Cities and Society, v. 28, p. 108-117, 2017.

MEBRATU, D. Sustainability and sustainable development: historical and conceptual review. Environmental Impact Assessment Review, v. 18, n. 6, p. 493-520, 1998.

MEIJER, A.; BOLÍVAR, M. P. R. Governing the smart city: a review of the literature on smart urban governance. International Review of Administrative Sciences, v. 82, n. 2, p. 392-408, 2016.

MENDES, J. M. G. Dimensões da sustentabilidade. Revista das Faculdades Santa Cruz, v. 7, n. 2, p. 4960, 2009.

MERRIMAN, K. K.; SEN, S. Incenting managers toward the triple bottom line: An agency and social norm perspective. Human Resource Management, v. 51, n. 6, p. 851-871, 2012.

NEIROTTI, P. et al. Current trends in smart city initiatives: some stylised facts. Cities, v. 38, p. 25-36, 2014.

PAPA, R. et al. Smart and resilient cities: a systemic approach for developing cross-sectoral strategies in the face of climate change. TeMA Journal of Land Use, Mobility and Environment, v. 8, n. 1, p. 19-49, 2015. 
PEREIRA, J. V. I. Sustentabilidade: diferentes perspectivas, um objectivo comum. Economia Global e Gestão, v. 14, n. 1, p. 115-126, 2009.

PERRINI, F; TENCATI, A. Sustainability and stakeholder management: the need for new corporate performance evaluation and reporting systems. Business Strategy and the Environment, v. 15, n. 5, p. 296-308, 2006.

PETTICREW, M.; ROBERTS, H. Systematic reviews in the social sciences: a practical guide. Malden, MA: Blackwell, 2006.

PIRO, G. et al. Information centric services in smart cities. Journal of Systems and Software, v. 88, p. 169188, 2014.

PRESLEY, A.; MEADE, L.; SARKIS, J. A strategic sustainability justification methodology for organizational decisions: a reverse logistics illustration. International Journal of Production Research, v. 45, n. 18-19, p. 4595-4620, 2007.

ROCA, L. C.; SEARCY, C. An analysis of indicators disclosed in corporate sustainability reports. Journal of Cleaner Production, v. 20, n. 1, p. 103-118, 2012.

SÁNCHEZ, L. et al. Integration of utilities infrastructures in a future internet enabled smart city framework. Sensors, v. 13, n. 11, p. 14438-14465, 2013.

SCOPUS. About Scopus. Disponível em <https:// www.elsevier.com/solutions/scopus $>$. Acesso em 22 jul. 2016.

SENATORE, G. Storia della sostenibilità: dai limiti della crescita alla genesi dello sviluppo. Milano: FrancoAngeli, 2013.

STÅHLBRÖST, A. et al. Design of smart city systems from a privacy perspective. International Journal on WWW/Internet, v. 13, n. 1, p. 1-16, 2015.
STRATIGEA, A.; PAPADOPOULOU, C.; PANAGIOTOPOULOU, M. Tools and Technologies for Planning the Development of Smart Cities. Journal of Urban Technology, v. 22, n. 2, p. 43-62, 2015.

UNEP. Ecodevelopment and the Irrational Use of Resources. Environmental Policy and Law, v. 3, p. 183-185, 1977.

WAAGE, S. A. et al. Fitting together the building blocks for sustainability: a revised model for integrating ecological, social, and financial factors into business decision-making. Journal of Cleaner Production, v. 13, n. 12, p. 1145-1163, 2005.

WAGNER, M. The role of corporate sustainability performance for economic performance: a firm-level analysis of moderation effects. Ecological Economics, v. 69, n. 7, p. 1553-1560, 2010.

WCED. World Commission on Environment and Development. Our common future. Oxford: Oxford University Press, 1987.

WOLFSWINKEL, J. F.; FURTMUELLER, E.; WILDEROM, C. P. M. Using grounded theory as a method for rigorously reviewing literature. European Journal of Information Systems, v. 22, n. 1, p.45-55, 2013.

ZINK, K. J. Designing sustainable work systems: the need for a systems approach. Applied Ergonomics, v. 45, n. 1, p. 126-132, 2014.

ZU BIZARRETA， I.; SERAVALLI, A .; ARRIZABALAGA, S. Smart City Concept: what it is and what it should be. Journal of Urban Planning and Development, v. 142, n. 1, p. 04015005-1 - 040150058, 2015. 